\title{
DADOS NORMATIVOS PARA O TESTE DE FLUÊNCIA VERBAL CATEGORIA ANIMAIS EM NOSSO MEIO
}

\author{
SONIA M. DOZZI BRUCKI*, SUZANA M. FLEURY MALHEIROS** \\ IVAN H. OKAMOTO***, PAULO H.F. BERTOLUCCI****
}

\begin{abstract}
RESUMO - Objetivos: Avaliar o desempenho na fluência verbal ern nossa população e verificar a influência da idade e escolaridade. Métodos: Foram entrevistados 336 indivíduos sem queixas neurológicas ou psiquiátricas, através do Mini-Exame do Estado Mental (MEM) e geração de animais em um minuto. Para efeito comparativo e para verificação dos níveis de corte, foram examinados 65 indivíduos com quadro de perda cognitiva, acompanhados no ambulatório de Neurologia do Comportamento da EPM. Resultados: Na população "normal" tivemos média de 13,84 animais por minuto. Para os grupos de escolaridade: 11,92, para analfabetos; 12,82 , para individuos com até 4 anos incompletos; 13,45 , para os de 4 a 8 anos incompletos; 15,88 para os com 8 ou mais anos de escolaridade. Houve diferença significante entre eles $(p=0,0001)$. Para os grupos etários, tivemos médias de: 13,79 , para aqueles com idade inferior a 65 anos; 13.92 para os com idade igual ou superior a 65 anos (sem diferença estatística). Determinamos para esses grupos dois níveis de corte: 9 para indivíduos com até 8 anos de escolaridade com sensibilidade de $75 \%$ para analfabetos, $100 \%$ para baixa escolaridade, $87 \%$ para média escolaridade; e especificidade de $79 \%$ para analfabetos, $84 \%$ para baixa escolaridade, $88 \%$ para média escolaridade. Para o grupo de alta escolaridade o escore de corte foi de 13 , com sensibilidade de $86 \%$ e especificidade de $67 \%$. Conclusão: Devemos utilizar níveis diferenciados de corte no teste de fluência verbal, em nosso meio, considerando os efeitos da escolaridade sobre o desempenho neste teste.
\end{abstract}

PALAVRAS-CHAVE: fluência verbal, escolaridade, linguagem.

\section{Normative data: category verbal fluency}

Abstract - Objective: Evaluate the performance on verbal fluency (VF) in our population in a Brazilian sample checking the influence of age and literacy. Methods: 336 people without neurological or psychiatric complaints evaluated through Mini-Mental State Examination and VF (animals). For comparison, and to determine cut-off points, 65 people with cognitive loss followed at our clinic were also evaluated. Results: We found a mean of 13.8 animals in 1 minute, with the following distribution: illiterates, 11.9; up 4 years of education, 12.8; 4 to 7 years, $13.4 ; 8$ years or more, $15.8(p=0.0001)$. In relation to age the means were: up to 64 years, $13.7 ; 65$ years or more, 13.9. There was no difference between the two groups. The cut-off points were 9 for people under 8 years of education with a sensitivity of $75 \%$ for illiterates, $100 \%$ for low educational level (up 4 years), and $87 \%$ for middle level ( 4 to 7 years). The specificity was respectively $79 \%, 84 \%$, and $88 \%$. For the high educational level the mean was 13 with a sensitivity of $86 \%$ and specificity of $67 \%$. Conclusions: In the VF (animals) there is a significant influence of schooling and different cut-off points should be used.

KEY WORDS: educational status, verbal fluency, language

O teste de fluência verbal (FV), comumente, está inserido em baterias neuropsicológicas ou é utilizado isoladamente, tanto para estudo da linguagem ou mudança de estratégia, como para

Disciplina de Neurologia da Escola Paulista de Medicina (EPM) da Universidade Federal de São Paulo (UNIFESP). *Mestre em Neurologia; **Doutora em Neurologia; ***Mestrando em Neurologia; **** Professor Adjunto. Aceite: 4-novembro-1996

Dra. Sonia M. Dozzi Brucki - Disciplina de Neurologia, Escola Paulista de Medicina, UNIFESP - Rua Botucatu 740 - 040023-900 São Paulo SP - Brasil. 
estudos específicos de memória semântica. $\mathrm{O}$ teste de FV, categoria animais, faz parte do Boston Diagnostic Aphasia Examination (BDAE) ${ }^{5,8}$, também foi incorporado ao protocolo do CERAD (Consortium to Establish a Registry for Alzheimer Disease) ${ }^{22}$. Dados normativos por idade em indivíduos com escolaridade superior a 4 anos foram demonstrados por Osterweill et $\mathrm{al}^{25}$. $O$ teste de FV foi utilizado em diversos tipos de estudos, comparando indivíduos com lesōes cerebrais localizadas 3.9,15,18,27.31; em estudos com pacientes com doença de Alzheimer ${ }^{1,2,6,13,19,24}$, também em estudos com indivíduos com doença de Parkinson ${ }^{10,14,17,29} \mathrm{e}$ em outros tipos de afecçōes, como doença de Huntington ${ }^{12,32}$, esclerose múltipla ${ }^{2 x}$, alcoolistas ${ }^{7,11}$.

No estudo de normatização do teste ${ }^{5}$ a escolaridade variou de zero a 16 anos, sem comentários quanto à influência da escolaridade. Nossa população tem escolaridade heterogênea e possui grande número de indivíduos com baixa escolaridade $e$ analfabetos. Não há estudos em nosso meio comparando o efeito da escolaridade sobre o desempenho no teste de FV. No estudo de Nitrini et $\mathrm{al}^{24}$ os resultados são de indivíduos de escolaridade superior a 4 anos. Na literatura encontramos um estudo comparando analfabetos a indivíduos com escolaridade superior a dez anos, com diferença significante nos desempenhos dos dois grupos ${ }^{30}$. Deste modo, a finalidade deste estudo foi a de avaliar o desempenho de indivíduos, no nosso meio, no teste de FV e analisar a influência da idade e escolaridade sobre ele.

\section{PACIENTES E MÉTOdO}

Foram estudados 336 indivíduos que obedeciam aos critérios de exclusão: idade inferior a 15 anos; individuos com história de epilepsia, acidente vascular cerebral, neurocirurgias, além de queixas psiquiátricas; outra língua materna que não a portuguesa; déficit visual ou auditivo não corrigidos; indivíduos com acometimento das funçð̃es motoras, por causas ortopédicas ou reumatológicas, que prejudicassem o desempenho em itens que a exigissem. Para efeito comparativo, os mesmos testes foram aplicados em pacientes acompanhados no Setor de Doenças Degenerativas da Disciplina de Neurologia da UNIFESP, com quadros de déficit cognitivo de causas variadas e em indivíduos com escores inferiores aos preconizados por escolaridade, segundo Bertolucci et al' ${ }^{4}$, no Mini-Exame do Estado Mental. Este grupo de indivíduos foi denominado "não normal".

A populaçăo "normal" foi classificada quanto à idade em: jovens (J), individuos com até 64 anos e idosos (I), indivíduos com idade igual ou superior a 65 anos. Além disso, foi também dividida quanto à escolaridade alcançada em: analfabetos (AN); baixa escolaridade (B), um a quatro anos incompletos; média escolaridade (M), quatro a oito anos incompletos; $e$ alta escolaridade (AL) oito e mais anos.

Após comunicarmos aos indivíduos de que seriam submetidos a urn teste de memória e afastados os criterios de exclusäo, forarn realizados o Mini-Exame do Estado Mental (MEM) de acordo com a versão e normas em portuguêst e o teste de FV categoria animais. Neste último foi pedido ao paciente que recordasse tantos animais ou "bichos" quanto possível, no intervalo de 60 segundos.

Na análise estatística foram utilizados os testes de Qui-Quadrado, Mann-Whitney, Kruskall-Wallis e medidas de sensibilidade e especificidade.

\section{RESULTADOS}

Na análise demográfica nossa amostra "normal" distribuiu-se conforme a Tabela 1. Na Tabela 2 observamos a distribuição do grupo quanto à escolaridade e grupo etário.

Não houve diferença quanto à distribuição do sexo entre os grupos etários $(p=0,2965)$, tampouco entre os grupos de escolaridade ( $\mathrm{p}=0,3422$ ) (teste do Qui-Quadrado). Não houve diferença na distribuição dos grupos etários segundo os grupos de escolaridade $(p=0,241)$.

Quanto ao desempenho no teste de FV para o grupo de indivíduos normais tivemos a média de 13,84 (desvio padrão de 4,22 ). No sexo feminino a média foi $13,68(4,13)$ e no masculino de $14,03(4,32)$, sem diferença significante $(p=0,4978)$. Quanto ao grupo jovem a média foi $13,79 \mathrm{e}$ no idoso foi 13,92 ( $p=0,4865$ ). Na Tabela 3 demonstramos o desempenho dos indivíduos normais, por escolaridade, observando-se diferença significante entre os resultados $(p=0,0001)$. Analisando- 
Tabela 1. Dados demográficos divididos por sexo, idade (em anos), grupo etário, escolaridade (em anos), grupo de escolaridade na população normal.

\begin{tabular}{ll}
\hline Sexo (\%) & $180(53,7 \%)$ \\
feminino & $156(46,42 \%)$ \\
masculino & \\
Idade (anos) & $55,26(18,73)$ \\
média (DP) & 57 \\
mediana & 15 \\
mínima & 91 \\
máxima & 76 \\
amplitude & \\
Grupo etário (\%) & $201(59,28 \%)$ \\
jovem & $135(40,18 \%)$ \\
idoso & \\
Escolaridade (anos) & $57(13,99 \%)$ \\
média (DP) & $59(14,58 \%)$ \\
mediana & $142(42,26 \%)$ \\
mínima & $48,38)$ \\
máxima & 0 \\
amplitude & 20 \\
Grupo de escolaridade $(\%)$ & \\
analfabeto & 20 \\
baixa & \\
média & \\
alta & \\
\hline & \\
\hline
\end{tabular}

se separadamente, por grupo etário, o desempenho entre as escolaridades, observamos que entre os grupos jovem e idoso mantém-se a mesma diferença estatisticamente significante. $O$ que não ocorre na análise dentro de cada nível de escolaridade, por grupo etário.

Na amostra "não normal" obtivemos a média de 7,35 animais em 60 segundos. A idade não teve influência sobre os escores no teste de $\mathrm{FV}(\mathrm{p}=0,2734)$, porém também neste grupo ocorreu diferença significante no desempenho no teste, entre as três escolaridades $(p=0,0159)$.

Deste modo tentamos determinar níveis de corte diferenciados para cada escolaridade. A escolha do nível de corte foi determinada pela soma de maior valor das sensibilidade e especificidade $\mathrm{em}$ vários escores. Para os analfabetos essa soma seria maior para o escore 7 , porém preferimos o escore 9 como corte para minimizar a utilização de vários valores. Como a soma das sensibilidade e especificidade nos grupos de baixa e média escolaridades é maior para o escore 9, esse foi o adotado também para os analfabetos. Neste grupo esse corte, embora não sendo o da maior soma, é o de maior sensibilidade. Também com o escore de 9, temos o maior valor preditivo negativo entre os escores nestas três escolaridades. Para a alta escolaridade o nivel de corte escolhido foi o de 13 animais, alcançando a maior soma de sensibilidade e especificidade, com o maior nível de sensibilidade e valor preditivo negativo. $\mathrm{Na}$ Tabela 4 temos os níveis de sensibilidade e especificidade para os escores e os valores preditivos para os níveis de corte.

Portanto, os níveis de corte obtidos foram: 9 para analfabetos, baixa e média escolaridades; e 13 para alta escolaridade.

\section{DISCUSSÃO}

Utilizamos o teste de fluência verbal por categoria semântica (categoria animais). A escolha do tipo de teste foi feita a partir da suposição de que, numa população com nível educacional heterogêneo, um teste de categoria semântica fosse menos vulnerável aos efeitos da escolaridade do que um teste de geraçāo de palavras iniciadas por letras pré-determinadas, que exigiria um vocabulário mais extenso do que o simples conhecimento de categorias semânticas bem definidas e comuns. Além do que este tipo de teste seria o melhor na deteç̧ão e diferenciaçăo entre índivíduos normais e com comprometimento cognitivo ${ }^{16,21,26}$. 
Tabela 2. Distribuição dos individuos do grupo "normal" segundo o grupo de escolaridade e grupo etário

\begin{tabular}{llll}
\hline Escolaridade & \multicolumn{2}{c}{ Grupo etário } \\
& jovem-n(\%) & idoso-n(\%) & total \\
\cline { 2 - 4 } analfabetos & $25(12,4 \%)$ & $22(16,3 \%)$ & 47 \\
baixa & $25(12,4 \%)$ & $24(17,8 \%)$ & 49 \\
média & $86(42,8 \%)$ & $56(41,5 \%)$ & 142 \\
alta & $65(32,35 \%)$ & $33(24,4 \%)$ & 98 \\
total & $201(100 \%)$ & $135(100 \%)$ & 336 \\
\hline
\end{tabular}

Teste do Qui-Quadrado $(p=0,241)$

Tabela 3. Comparação dos escores dos individuos "normais", por escolaridade, pelo teste de Kraskal-Wallis.

\begin{tabular}{llllll}
\hline & AN & B & M & AL & \\
\hline Fluência verbal & & & & & \\
média (DP) & $11,92(3,24)$ & $12,82(3,72)$ & $13,45(3,92)$ & $15,88(4,56)$ & $\mathrm{p}=0,0001$ \\
mediana & 11 & 13 & 13 & 16 & \\
mínimo & 5 & 4 & 5 & 7 & 29 \\
máximo & 18 & 24 & 23 & & \\
MEM & & & & $27,97(1,56)$ & $\mathrm{p}=0,0001$ \\
média (DP) & $20,72(3,1)$ & $24,33(3,21)$ & $25,52(2,82)$ & 28 & \\
mediana & 21 & 25 & 26 & 28 & \\
\hline
\end{tabular}

Não observamos diferença quanto ao desempenho no teste, por grupo etário. Rosselli e Ardila $^{30}$ não observaram interação da idade com o desempenho, tanto no grupo de analfabetos quanto no de alta escolaridade, no teste por categoria animais. Nitrini ${ }^{23}$ não encontrou decréscimo na produção de animais com o envelhecimento: sua amostra dividia-se por décadas, sendo que até a oitava década não houve diminuição significante.

Nosso estudo demonstrou que a escolaridade tem influência significante sobre o desempenho no teste. No estudo de Unverszagt et $\mathrm{al}^{33}$, com escolaridade variando de zero a 16 anos, não foi observada a influência da escolaridade, talvez devendo-se ao pequeno tamanho da amostra (83 indivíduos) e também à sua escolaridade média, que foi de 9,9 anos, superior à nossa de 5,66 anos. No estudo normativo de Borod et $\mathrm{al}^{5}$ as escolaridades variaram de zero a 16 anos, porém o grupo de zero a oito anos era composto por apenas 11 indivíduos. A média nesse estudo foi de 22,5 animais.

Também para Welsh et $\mathrm{al}^{34}$ não ocorre influência da educação sobre os escores; dividia-se em indivíduos com escolaridades inferior ou superior a 12 anos. Foram excluídos os indivíduos com escolaridade de zero a quatro anos, no estudo de Osterweil et al $^{23}$, em que não se observou influência da escolaridade. Para Rosselli e Ardila ${ }^{30}$ ocorreu diferença significante entre o desempenho de analfabetos e indivíduos com escolaridade superior a dez anos, sendo que a média de seus analfabetos assemelhou-se à nossa.

Utilizando o valor de corte preconizado de $12^{5,20}$, teríamos para os analfabetos uma sensibilidade de $75 \%$ e especificidade de $38 \%$; para baixa escolaridade, $100 \%$ de sensibilidade e 
Tabela 4. Niveis de sensibilidade e especificidade por escolaridade dos escores e valores preditivos positivos e negativos para os niveis de corte.

\begin{tabular}{|c|c|c|c|c|c|}
\hline Escolaridade & Escore & Sensibilidade & Especificidade & $\begin{array}{l}\text { Valor preditivo } \\
\text { positivo }\end{array}$ & $\begin{array}{c}\text { valor preditivo } \\
\text { negativo }\end{array}$ \\
\hline \multirow[t]{5}{*}{ analfabetos } & 7 & $62 \%$ & $96 \%$ & & \\
\hline & $8(P 10)$ & $62 \%$ & $89 \%$ & & \\
\hline & 9 & $75 \%$ & $79 \%$ & 0,37 & 0,95 \\
\hline & $10(P 25)$ & $75 \%$ & $62 \%$ & & \\
\hline & 11 & $75 \%$ & $47 \%$ & & \\
\hline \multirow[t]{5}{*}{ baixa } & 7 & $62 \%$ & $75 \%$ & & \\
\hline & $8(P 10)$ & $92 \%$ & $82 \%$ & & \\
\hline & 9 & $100 \%$ & $84 \%$ & 0,68 & 1,00 \\
\hline & 10 & $100 \%$ & $78 \%$ & & \\
\hline & $11(\mathrm{P} 25)$ & $100 \%$ & $61 \%$ & & \\
\hline \multirow[t]{4}{*}{ média } & 8 & $82 \%$ & $92 \%$ & & \\
\hline & $9(\mathrm{P} 10)$ & $87 \%$ & $88 \%$ & 0,54 & 0,98 \\
\hline & $10(\mathrm{P} 25)$ & $91 \%$ & $72 \%$ & & \\
\hline & 11 & $100 \%$ & $60 \%$ & & \\
\hline \multirow[t]{6}{*}{ alta } & 8 & $36 \%$ & $96 \%$ & & \\
\hline & $10(\mathrm{P} 10)$ & $54 \%$ & $90 \%$ & & \\
\hline & 11 & $68 \%$ & $84 \%$ & & \\
\hline & $12(\mathrm{P} 25)$ & $77 \%$ & $74 \%$ & & \\
\hline & 13 & $86 \%$ & $67 \%$ & 0,37 & 0,96 \\
\hline & 14 & $91 \%$ & $42 \%$ & & \\
\hline
\end{tabular}

P10, percentil 10; P25, percentil 25.

especificidade de 51\%; para os de média, $100 \%$ de sensibilidade e $55 \%$ de especificidade, e para os de alta escolaridade $77 \%$ de sensibilidade e $74 \%$ de especificidade. Comparando nossos resultados aos de Nitrini et $\mathrm{al}^{24}$, realizado em nosso meio, observamos que nossa média (13,84 animais) foi inferior à encontrada por esses autores $(17,93)$, possivelmente devido à inclusão, em nossa amostra, de indivíduos analfabetos e de escolaridade inferior a quatro anos, excluídos por esses autores. Considerando o corte de 13 destes autores e utilizando-o em nossa amostra "normal", teriamos sensibilidade de $90 \%$ e especificidade de apenas $50 \%$. Considerando nossos indivíduos analfabetos, uma sensibilidade de $100 \%$, porém com especificidade de $34 \%$ e na baixa e média escolaridades, teriamos sensibilidade de $100 \%$ e especificidade de $45,5 \%$. Para esse corte de 13 , apenas $50,2 \%$ dos nossos indivíduos "normais" estariam acima deste nível.

Deste modo, adotamos para uma população de escolaridade heterogênea, dois níveis de corte: para aqueles desprovidos de qualquer escolaridade e até oito anos incompletos, o nível de corte de 9, e para aqueles com oito ou mais anos de escolaridade, o nível de 13 animais em um minuto. Concluímos que não é possível a extrapolaçăo de dados da literatura, como nível de corte, para nossa população. 


\section{REFERÊNCIAS}

1. Appell J, Kertesz A, Fisman M. A study of language functioning in Alzheimer disease. Brain Lang 1982;17:73-91.

2. Becker JT, Huff FJ, Nebes RD, Holland A, Boller F. Neuropsychological function in Alzheimer's disease: pattern of impairment and rates progression. Arch Neurol 1988;45:263-268.

3. Benton AL. Differential behavioral effects in frontal lobe disease. Neuropsychologia 1968;6:53-60.

4. Bertolucci PHF, Brucki SMD, Campacci SR, Juliano Y. O Mini-Exame do Estado Mental em uma populaçăo geral: impacto da escolaridade. Arq Neuropsiquiatr 1994;52:1-7.

5. Borod JC, Goodglass H, Kaplan E. Normative data on the Boston Diagnostic Aphasia Examination, Parietal Lobe Battery, and the Boston Naming Test. J Clin Neuropsychol 1980;2:209-215.

6. Cormier P, Margison JA, Fisk JD. Contribution of perceptual and lexical semantic errors to the naming impairments in Alzheimer's disease. Percept Mot Skills 1991;73:175-183.

7. Cutting J. Specific psychological deficits in alcoholism. Br J Psychiarry 1978;133:119-122.

8. Goodglass H, Kaplan E. The assessment of aphasia and related disorders. Ed2. Philadelphia: Lea \& Febinger, 1983:14,40-41.

9. Grossman M. A bird is a bird: making reference within and without superordinate categories. Brain Lang 1981;12:313-331.

10. Gurd JM, Ward CD. Retrieval from semantic and letter-initial categories in patients with Parkinson's disease. Neuropsychologia 1989;27:743-746.

11. Hewett LJ, Nixon SJ, Glenn SW, Parsons OA. Verbal fluency deficits in female alcoholics. J Clin Psychol 1991;47:716-720.

12. Hodges JR, Salmon DP, Butters N. Differential impaiment of semantic and episodic memory in Alzheimer's and Huntington's diseases: a controlled prospective study. I Neurol Neurosurg Psychiatry 1990;53:1089-1095.

13. Hodges JR. Salmon DP, Butters N. Semantic memory impairment in Alzheimer's disease: failure of access or degraded knowledge. Neuropsychologia 1992;30:301-314.

14. Jacobs DM, Marder K, Côté L, Sano M, Stern Y, Mayeux R. Neuropsychological characteristics of preclinical dementia in Parkinson's disease. Neurology 1995;45:1691-1696.

15. Joanette $Y$, Goulet $P$, Le Dorze G. Impaired word naming in right-brain damage right-handers: error types and time-course analyses. Brain Lang 1988:34:54-64.

16. Kertesz A, Clydesdale S. Neuropsychological deficits in vascular dementia vs Alzheimer's disease: frontal lobe deficits prominent in vascular dementia Arch Neurol 1994;51:1226-1231.

17. Levin BE, LlabreMM, Weiner WJ. Cognitive impairments associated with early Parkinson's disease. Neurology 1989:39:557-561.

18. Lucchelli F, DeRenzi E. Proper name anomia. Cortex 1992;28:221-230.

19. Masur DM, Sliwinski M, Lipton RB, Blau AD, Crystal HA. Neuropsychological prediction of dementia and the absence of dementia and the absence of dementia in healthy elderly persons. Neurology 1994:44:1427-1432.

20. Mesulam M-M. Principles of behavioral neurology. Philadelphia F.A. Davis. 1985:198.

21. Monsch AU, Bondi MW, Butters N, Salmon DP, Katzman R, Thal LJ. Comparisons of verbal fluency tasks in the detection of dementia of the Alzheimer type. Arch Neurol 1992;49:1253-1258.

22. Morris JC, Heyman A, Mohs RC, Hughes JP, vanBelle G, Fillenbaum G, Mellits ED, Clark C, and the CERAD investigators. The Consortium to Establish a Registry for Alzheimer's disease (CERAD); Part 1. Clinical and neuropsychological assessment of Alzheimer's disease. Neurology 1989;39:1159-1165.

23. Nitrini R. In Monografias em geriatria III. Envelhecimento do sistema nervoso e a dor no idoso. São Paulo:FMUSP,1996:64-65.

24. Nitrini R, Lefeve BH, Mathias SC, Caramelli P, Carrilho PEM, Sauaia N, Massad E, Takiguti C, Silva IO, Porto CS, Magila MC, Scaff M. Testes neuropsicológicos de aplicação simples para o diagnóstico de demência. Arq Neuropsiquiatr 1994;52:457-465.

25. Osterweill D, Mulford $P$, Syndulko K, Martin M. Cognitive function in old and very old residents of a residential facility: relationship to age, education, and dementia. J Am Geriatr Soc 1994;42:766-773.

26. Pasquier F, Lebert F, Grymonprez L, Petit $\mathrm{H}$. Verbal fluency in dementia of frontal lobe type and dementia of Alzheimer type.J Neurol Neurosurg Psychiatry 1995;58:81-84.

27. Perret $E$. The left frontal lobe of man and the supression of habitual responses in verbal categorical behaviour. Neuropsychologia 1974;12:323-330.

28. Pozzilli C, Bastianello S, Padovani A, Passafiume D, Millefionini E, Bozzao L, Fieschi C. Anterior corpus callosum atrophy and verbal fluency in multiple sclerosis. Cortex 1991;27:441-445.

29. Raskin SA, Sliwinski M, Borod JC. Clustering strategies on tasks of verbal fluency in Parkinson's disease. Neuropsychologia 1992;30:95-99.

30. Rosselli M, Ardila A. Neuropsychological assessment in illiterates: II. Language and praxic abilities. Brain Cogn 1990;12:281-296.

31. Schneiderman EI. A linguistic deficit resulting from right-bemisphere damage. Brain Lang 1988;34:38-53

32. Troster AI, Salmon DP, McCullough D, Butters N. A comparison of the category fluency deficits associated with Alzheimer's and Huntington's disease. Brain Lang 1989;37:500-513.

33. Unverszagt FW, Hui S, Farlow MR, Hall K, Hendrie HC.Cognitive decline is greater in dementia patients with high education. Neurology 1995;45(Suppl 4): 272.

34. Welsh KA, Butters N, Mohs RC, Beekly D, Edland S, Fillenbaum G, Heyman A. The Consortium to Establish a Registry for Alzheimer's Disease (CERAD): Part V. A normative study of the neuropsychological battery. Neurology 1994;44:609-614. 